\title{
EFFECTS OF ALLOPURINOL ON IRON STORAGE IN THE RAT
}

\author{
BY \\ L. W. POWELL* \\ From the Medical Professorial Unit, University of Queensland, Brisbane, Australia
}

Iron is stored in the liver and reticulo-endothelial system in the oxidized or ferric state and is bound to protein as ferritin or haemosiderin. Ferrous iron is less tightly bound to protein than is the ferric form. The mechanism of release of iron from ferritin is uncertain but there is some evidence that the xanthine oxidase system is of physiological importance in this step (Fig. 1). According to this hypothesis the reduction of ferric to ferrous ferritin occurs in association with the oxidation of xanthine and hypoxanthine to uric acid, xanthine oxidase acting as an electron donor in the reduction reaction and an electron acceptor in theoxidation reaction (Fridovich and Handler, 1958a, b).

Forrous Ferritin

Fig. 1.-Hypothetical role of xanthine oxidase in reduction of ferric to ferrous ferritin and oxidation of xanthine to uric acid (reproduced from Lancet (1966) 1, 239, by permission of the Editor).

The main evidence for a role of xanthine oxidase in the mobilization of storage iron comes from experimental observations. In a study of the mechanism of the reduction of ferritin iron, Green and Mazur (1957) found that ferritin was reduced by xanthine oxidase in vitro and small amounts of iron were liberated. Mazur, Green, Saha, and Carleton (1958) reported that, in dogs, the mobilization of storage iron induced by haemorrhagic shock was accompanied by a rise in serum uric acid (SUA) levels. They also demonstrated that the infusion of xanthine and hypoxanthine resulted in a rise, not only in SUA levels, but also in serum iron levels. Cheney and Finch (1960) showed that the parenteral administration of xanthine oxidase substrates in experimental animals was followed by a 50 to 100 per cent. rise in iron absorption. Mazur and Carleton (1965) demonstrated that the very high ferritin content of

* Present address: c/o Medical Professorial Unit, Royal Brisbane Hospital, Brisbane, Australia. the liver of the new born rat was associated with an absence of hepatic xanthine oxidase activity. Within 6 to 10 days after birth hepatic xanthine oxidase $\vec{\omega}$ activity increased significantly coincident with ao marked decrease in ferritin iron. This observation $\frac{0}{2}$ could be of considerable biological significance since it is immediately after birth that the need for iron for haemoglobin synthesis increases rapidly and the maternal source of iron is no longer present. Thus? with the appearance of xanthine oxidase in the liver ferritin iron is released for transport to the boneo marrow for incorporation into haemoglobin.

Unfortunately this hypothesis does not explain alf the known or observed facts, and other experimentalwork has suggested that the relationship between xanthine oxidase and hepatic iron metabolism is an incidental one. For example, Strohmeyer, Gre berg, Moore, and Chalmers (1961) produced changesD in iron metabolism in rats by hypoxia but they faied to demonstrate concomitant changes in hepatiog

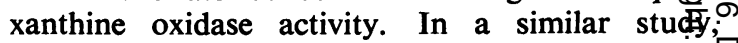
Strohmeyer, Miller, Scarlata, Moore, Greenberg, and Chalmers (1964) found that xanthine loading had nos effect on the release of tissue iron or on iron absorp-0 tion in rats. Kinney, Kaufman, and Klavins (1961) produced a reduction in hepatic xanthine oxidase activity with ethionine and sodium tungstate, bus were unable to demonstrate any concomitant altera- tion in hepatic storage iron or iron absorption? Ayvazian (1964) reported a patient with both xan? thinuria and haemochromatosis and a striking de-w crease in hepatic xanthine oxidase activity. He drew attention to the possible role of xanthine oxidase deficiency in idiopathic haemochromatosis, but के search of patients with haemochromatosis by ouro selves and others has failed to reveal other similar cases or any abnormality in the SUA level. We alsor know of one patient with haemochromatosis and symptomatic gout (Price Evans, 1966)!

Because of the possible inverse relationshing between hepatic xanthine oxidase activity and hepatiç iron storage, we considered it important to watch for changes in iron metabolism following the therapeutie inhibition of xanthine oxidase. It is my task tq outline the results of an experiment designed to stud 
the effect of allopurinol on hepatic iron storage in rats, and Dr. Emmerson will then give the results of clinical studies.

\section{Method}

We had four groups of male Wistar rats aged 6 weeks. Each group was given one of the following diets and tap water ad libitum:

\begin{tabular}{|c|c|c|}
\hline Group & No. of Rats & Diet \\
\hline A & 20 & Laboratory meal \\
\hline B & 12 & $\begin{array}{l}\text { Laboratory meal }+5 \text { per cent. ferric } \\
\text { ammonium citrate }\end{array}$ \\
\hline C & 6 & $\begin{array}{l}\text { Laboratory meal }+0.01 \text { per cent. } \\
\text { allopurinol }\end{array}$ \\
\hline D & 6 & $\begin{array}{l}\text { Laboratory meal }+0.01 \text { per cent. } \\
\text { allopurinol }+5 \text { per cent. ferric } \\
\text { ammonium citrate }\end{array}$ \\
\hline
\end{tabular}

0.01 per cent. allopurinol is equivalent to approximately $10 \mathrm{mg} . / \mathrm{kg}$. body weight/day for each animal, and this is equivalent to about $300 \mathrm{mg} . / \mathrm{kg}$. for an adult human. All animals were kept under the same environmental conditions and were weighed weekly. Diets were replenished and weighed daily. The animals were killed at intervals and specimens of liver obtained for microscopy and chemical estimation of iron content.

\section{Results}

Fig. 2 shows the regression lines for hepatic iron concentration with time. There was a significant difference between the regression coefficients for rats receiving meal + allopurinol (Group $C$ ) and rats receiving laboratory meal alone (Group A). This

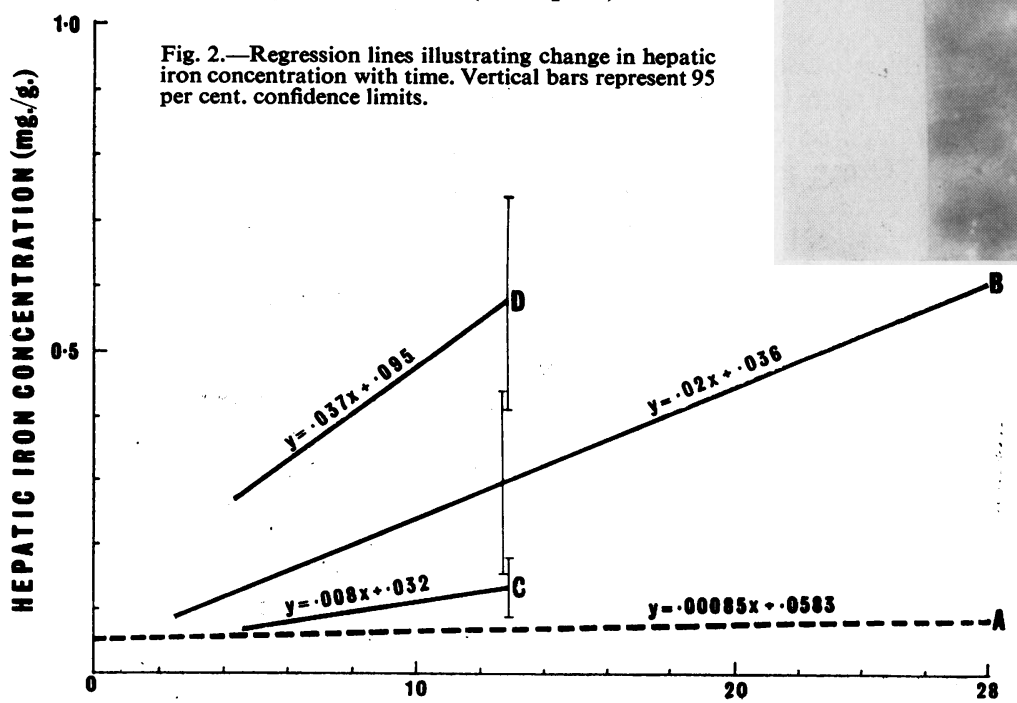

TIME (WKS) difference was significant at the 1 per cent. level of probability. The rise in hepatic iron concentration with time was steeper for rats receiving added allopurinol and iron (Group D) than for rats receiving added iron only (Group B), but the regression coefficients did not differ significantly. The presence of allopurinol did not affect food consumption or growth.

Fig. 3 shows the distribution and severity of the

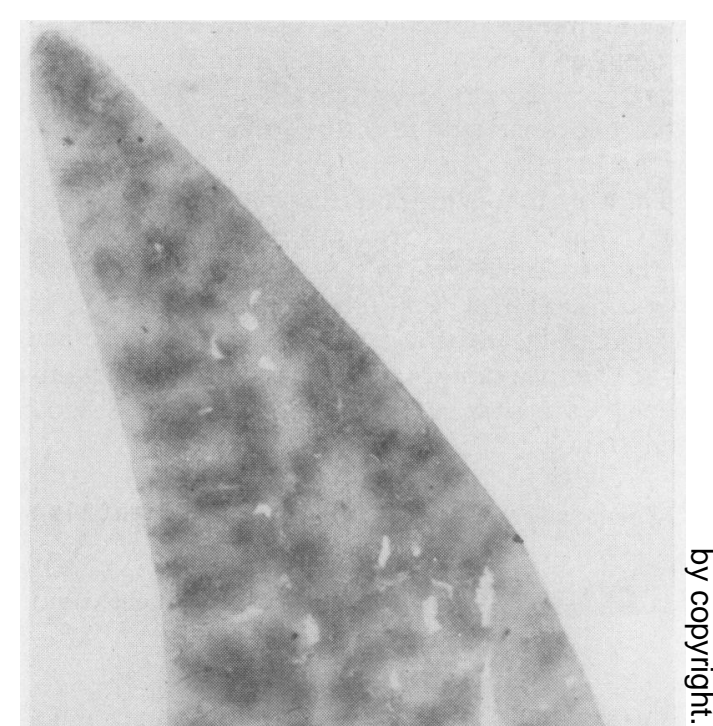

Fig. 3.-Section of liver of rat treated with laboratory meal diet with added iron and allopurinol for 13 weeks. There is a heavy deposition of iron in a periportal distribution. Perls' stain. $\times 12$. 
iron deposition in a histological section from the liver of a rat fed laboratory meal + iron + allopurinol (Group D) for 13 weeks. The accumulation of storage iron is seen in a periportal distribution. Comparable sections from rats fed meal +5 per cent. ferric ammonium citrate (Group B) for 13 to 28 weeks showed lesser c'egrees of haemosiderosis, but there was considerable variation from one animal to another. The concentrations of iron in the livers of rats fed laboratory meal only and laboratory meal + allopurinol (Groups A and C) did not reach the levels necessary for histochemical staining (i.e. $0 \cdot 2$ to $0.25 \mathrm{mg} . / \mathrm{kg}$.).

\section{Summary}

Xanthine oxidase activity is associated under some experimental conditions with the release of storage iron from the liver. In this animal experiment a group of rats fed allopurinol had more iron in the liver than did control animals. It should be emphasized that the numbers of animals were small (only six rats in each allopurinol-treated group), but the results indicate a need for further investigation into the effect of xanthine oxidase inhibition on iron storage and in particular for careful observation of iron metabolism in patients receiving allopurinol. 